\title{
A Smart Home Design Based on Ethernet
}

\author{
Wafaa Mustafa Abduallah르, Riyadh Zaghlool Mahmood² and Dakhaz Mustafa Abdullah ${ }^{1}$ \\ 1Department of Computer Science, College of Computer \& Information Technology, Nawroz University, Duhok, \\ Kurdistan Region - Iraq \\ 2Department of Software Engineering, College of Computer Science and Mathematics, Mosul University, Mosul - Iraq
}

\begin{abstract}
People are getting more concerned to protect their houses from unauthorized people, the status of the house environment like temperature, humidity, gas leakage and fire. The proposed system can monitor a house by use of sensors that are integrated with an Arduino microcontroller and an Ethernet shield unit. Notification message is used to alert users via mobile phone when a possible intrusion occurs or any abnormal events occurs in the Home environment. Nowadays, everyone is almost using mobile phone so via using this system, the users will not have to carry additional devices to monitor their house. This system is designed using modularity to become a flexible system that can be add more sensors without change the whole system, only add some sensors to increase systems functionality. So this system is a modular home security system using Ethernet that uses the internet via Blynk application function to initiate the communication between the system and the user.
\end{abstract}

KEY WORDS: Ethernet, Arduino, Microcontroller, Sensors, Blynk.

\section{INTRODUCTION}

Recently constructed buildings tend to be "intelligent" to enhance the convenience and safety of occupants. The concept of home automation and its safety has been around since late 1970s. But, with the advancement of technology, the expectation from home has changed a lot and so have the idea of home automation and its security systems (Lee \& Lee, 2004). So, looking at different home automation systems over time, they have always tried to provide efficient, convenient and safe ways for home inhabitants to access their homes (Zhai et. al, 2014), (Chitnis, Deshpande, \& Shaligram, 2016). At present, information technology is slowly changing people's traditional way of life and work; smart home has started to enter people's lives. Smart home not only has the traditional residential function, but also allows users to use more convenient means to manage home devices.

Academic Journal of Nawroz University (AJNU)

Volume 6, No 3(2017), 5 pages

Received 1 June 2017; Accepted 13 August 2017

Regular research paper: Published 15 August 2017

Corresponding author's e-mail: heevy9@yahoo.com

Copyright (C2017 Wafaa Mustafa Abduallah

This is an open access article distributed under the Creative

Commons Attribution License.
Users at anytime and anywhere outside can switch or adjust the indoor equipment through remote control. Smart home can also realize home energy management, and greatly improve the quality of people's lives (Teymourzadeh, Ahmed, Chan, \& Hoong, 2013), (Wu et. al, 2007), (Lee \& Lee, 2004).

At present, restricted by the existing technical conditions and economic costs, the smart home system is expensive and not very popular, resulting in a very high position but a narrow target market. In addition, a variety of smart home products on the market are lack of uniform technical standards and practicality, and the operation is complicated. Moreover, they cannot realize effective home energy management. The top priority is to design a simple, effective and low-cost smart home system, which will play a very big role in the application and promotion of smart home (Vanus et.al, 2016), (Piyare, 2013), (Lee \& Lee, 2004).

This paper is organized as follows: Section 2 discusses the related works, including a review of the modules that have been proposed by other researchers. Section 3 describes the design of the system with explaining the hardware components. Section 4 provides a clarification of software framework for the system. 5 provides a conclusion. 


\section{REVIEW OF SMART HOME MODELS}

Home automation or Smart Homes can be described as introduction of technology within the home environment to provide comfort, convenience, energy efficiency and security to its occupants (Piyare, 2013). Adding intelligence to home environment can provide increased quality of life for the elderly and disabled people who might otherwise require caregivers or institutional care. There has been a significant increase in home automation in recent years due to higher affordability and advancement in Smart phones and tablets which allows vast connectivity (Liu, 2013).

Many research have been conducted for designing smart home systems for intelligent remote monitoring and controlling home applications and most of these research carried out to fit some categories such as Internet based Monitoring using Servers, GPRS modems, etc. with various approaches (Piyare, 2013), (Gao, ,Wang, Zhou \& Zhang, 2016), (Han, et. al 2014), (Shewale, 2015), GSM-GPRS protocols using module individually or in combination with Internet Technologies (Zhang, Zou, \& Huang, 2013), (Teymourzadeh, Ahmed, Chan, \& Hoong, 2013), (Alheraish, 2004), Wireless Sensor Networks especially for Monitoring using Bluetooth, Wi-Fi, etc. and Biometric has various wide range applications like Home Automation, Home Security Systems, Biomedical Applications, Health Monitoring, Agriculture, etc (Butt, Khanam, Khan, \& Khiyal, 2010). More precisely, (Piyare, 2013) presented a low cost and flexible home control and monitoring system using an embedded micro-web server, with IP connectivity for accessing and controlling devices and appliances remotely using Android based Smart phone app. The proposed system does not require a dedicated server PC with respect to similar systems and offers a novel communication protocol to monitor and control the home environment with more than just the switching functionality.

(Teymourzadeh, Ahmed, Chan, \& Hoong, 2013) investigated the potential of implementing 'Full Home Control', which is the aim of the Home Automation Systems in near future. The analysis and implementation of the home automation technology using Global System for Mobile Communication (GSM) modem to control home appliances such as light, conditional system, and security system via Short Message Service (SMS) text messages is presented in this paper. The proposed research work is focused on functionality of the GSM protocol, which allows the user to control the target system away from residential using the frequency bandwidths. The concept of serial communication and AT-commands has been applied towards development of the smart GSM-based home automation system. Home owners will be able to receive feedback status of any home appliances under control whether switched on or off remotely from their mobile phones. PIC16F887 microcontroller with the integration of GSM provides the smart automated house system with the desired baud rate of 9600 bps.

(Butt, Khanam, Khan, \& Khiyal, 2010) work firstly tried to understand the voice of user then to control the home appliances through voice call and lastly to find out intrusion in the house. They also provided security and proposed SMS based system for controlling of home appliances remotely when the user is away from the place. The GSM Modem used in this paper allows to send and receive SMS to and from the system via RS232 serial port.

\section{DESIGN OF SMART HOME SYSTEM}

The process of designing and implementing this project require two mainly steps; the first step is about to combine all hardware components with Arduino Mega as shown in Fig. 1. While, the second part is about programming the Arduino Maga in order to be controlled by the mobile phone.

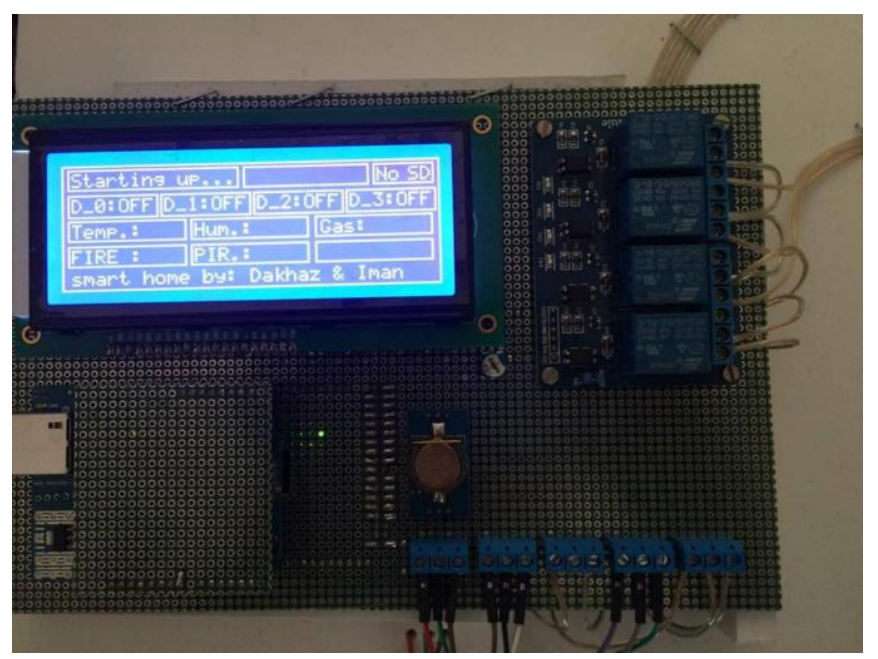

Fig. 1. The Implemented Arduino Mega with other Components of the proposed System.

\subsection{Hardware Framework}

The hardware part of the proposed system consists of seven parts where the whole six parts are connected with the Arduino part (which can be considered as a main board) in order to do a dedicated job as displayed in Fig. 2, these parts can be further explained through the following points: 


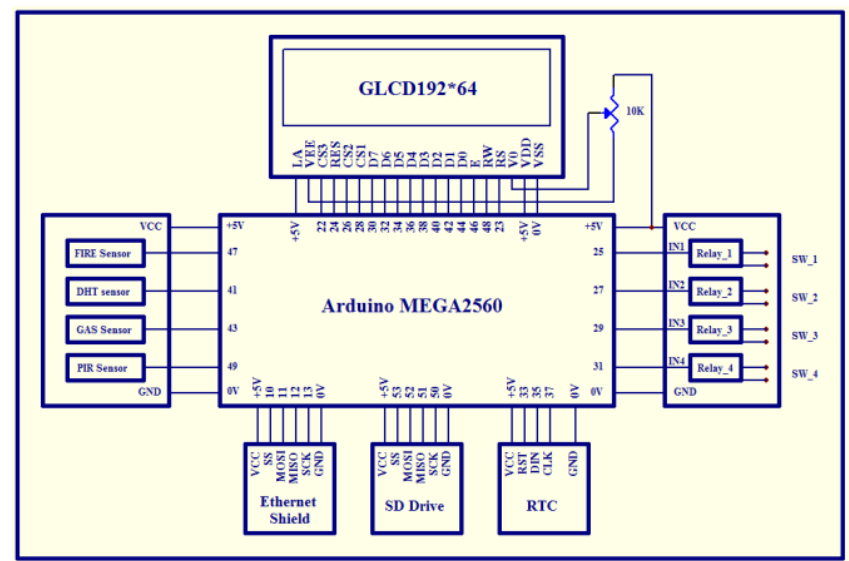

Fig. 2. Complete wiring diagram for the designed system

1. Arduino MEGA2560: Which is the main dominant of the components of the system and consists of a controlled accurate built in RAM, ROM and flash, as well as digital input and Output ports (54-PIN) and analogue (16-pin) that are used to connect the remaining parts of the system with Arduino according to their job, nature and type of signals that deals which can be either an input, director, digital or analog as shown in Fig. 3. This kind of dominant has been chosen for this project because of the urgent need to the relatively large numbers of pins, according to the system requirements.

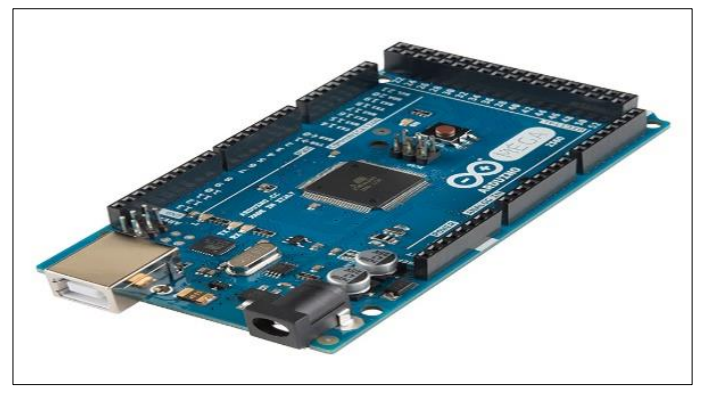

Fig. 3. Arduino MEGA2560

2. GLCD192*64: A graphic LCD (liquid crystal display) which is Graphical display with horizontal dimensions of 192 points and vertical points of 64 as displayed in Fig. 4. It is used to display the status of the system which are represented with the values of sensors and relays that are connected to the electrical appliances. In addition, to the Ethernet status, system clock and other futuristic purposes.

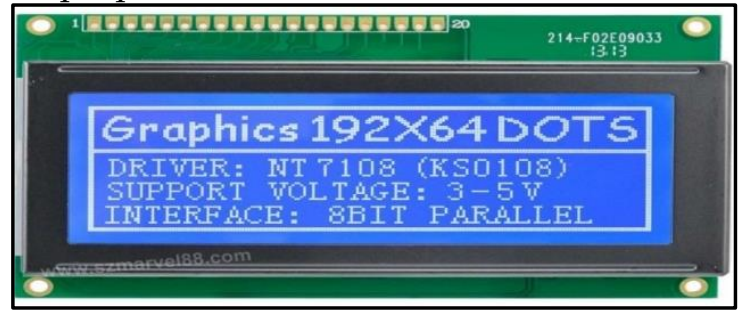

Fig.4. GLCD Screen
3. Sensors: The number of sensors in the proposed system is four, the first one is fire sensor that detects the presence of fire in the surrounding system environment, gas leak, temperature and humidity sensors as well as motion sensors to detect the entry of unauthorized persons to the home, which is intended to control the security, as displayed in Fig. 5.

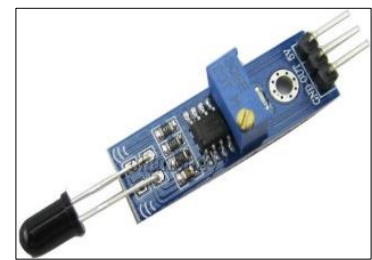

(a) Fire Sensor

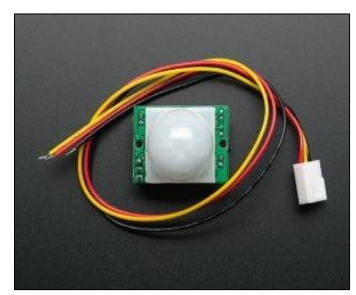

(c) PIR Sensor

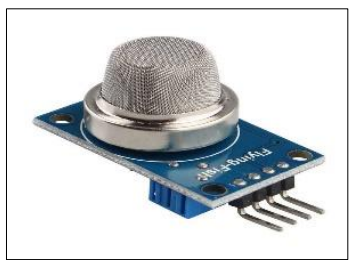

(b) Gas Sensor

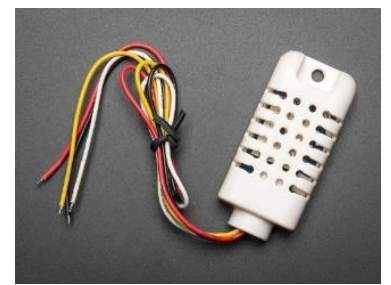

(d) temperature and humidity sensor
Fig. 5. The Sensors that have been adopted by the Proposed System.

4. Relays: The number of relays that are connected to the system are four that are used to turn on/off the remotely connected devices to it via the net and the application of the (Blynk). Usually these relays operate as electric keys to control any electrical device.

5. RTC: Real time clock is used to know the current time as well as saving time and date of any event occurred in the system to monitor the environment.

6. Ethernet Shield: it is a cover that used to connect the system with the Internet through (UTP Cable) to receive orders via the Internet that are sent by the user who's in charge of the system by the above application.

7. SD: Solid-state driver, used to store all the activities carried out by the system in terms of received orders and transmitted notifications to the user by date and time. It is possible to use a flash memory with size of 1 GB or more which can be considered as very large capacity relatively to the work of system.

\subsection{Software Framework}

Fig. 6 elaborated how the components that are connected with the Arduino Mega such as GLCD, the sensors and relays, RTC have been programmed. Firstly, the boarders and rectangles at the GLCD have been drawn in order to be used for displaying the text inside them. Then, 
function of Programming and Controlling Relays and Sensors (PCRS) is used to read and change the status of relays and sensors and consequently saving it to Secure Digital (SD) Card using function called (save_to_sd) as well as displaying it on the GLCD. Finally, sending notifications to the mobile when the status of the sensors is changed.

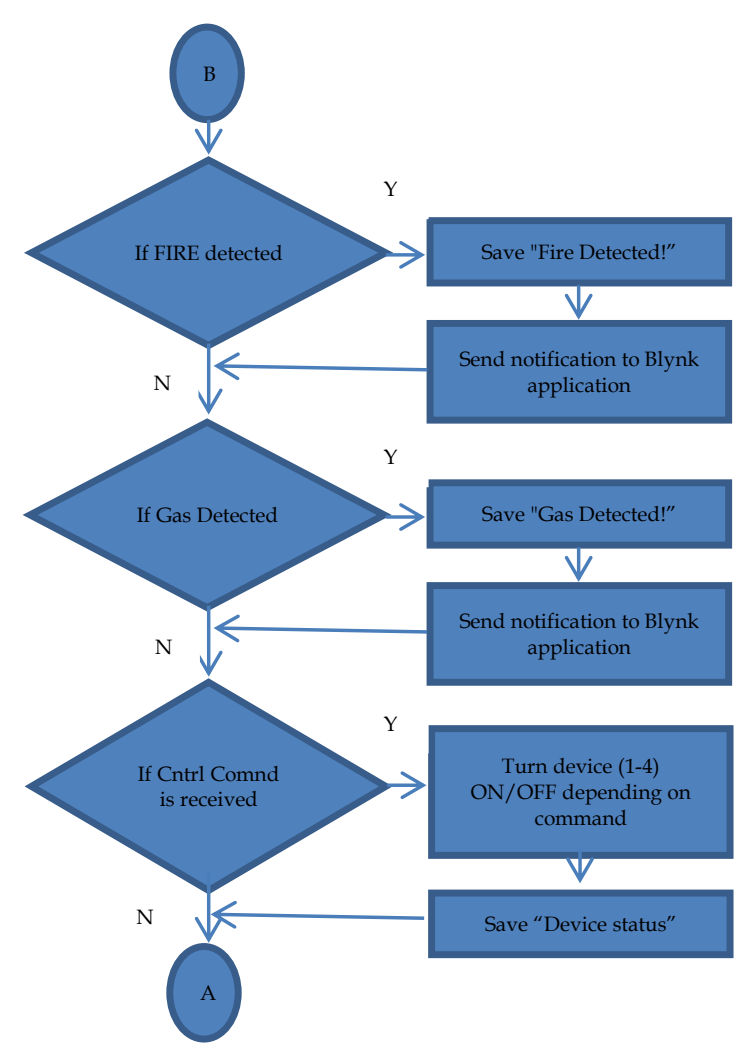

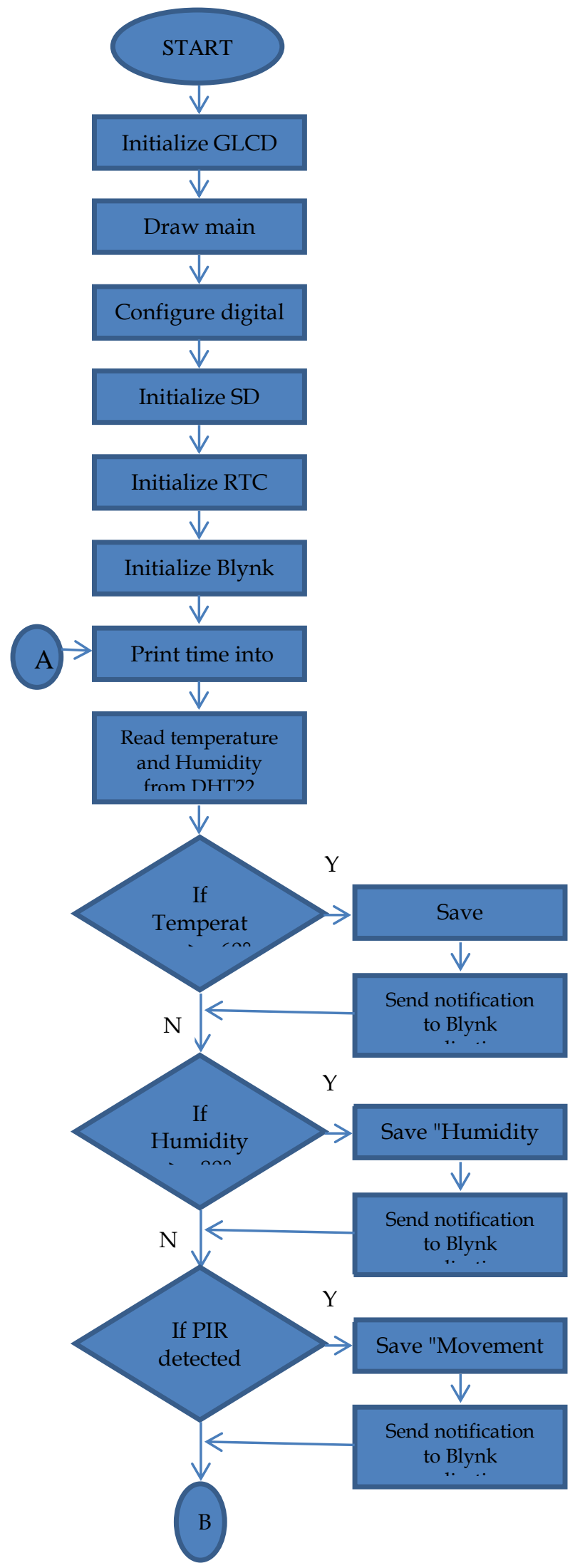

Fig 6. Complete Flowchart of the system 
More precisely, the steps of flowchart for the proposed system can be further explained as the following:

Step1: Initialize GLCD using its Arduino library by sending the actual control signal to internal registers before using it for printing characters and drawing boarders and shapes.

Step2: Drawing Main boarder including the places for displaying sensors value, Devices status and received messages.

Step3: Configuring digital input/output pins used for sensors, relays, GLCD display and communication pins between Arduino MEGA and Ethernet shield.

Step4: Initialize SD memory driver before reading/writing information from/to SD memory.

Step5: Initialize RTC (Real Time Clock) by configuring its pins using its Arduino library.

Step6: Initialize BLYNK application before connecting with the mobile phone to communicate with the device.

Step7: Printing the current time on its location in GLCD depends on the box on the GLCD.

Step8: Reading Temperature and Humidity values from corresponding sensors and printing their values on the GLCD.

Step9: If temperature is exceeds the threshold $(60 \mathrm{oC})$ then save the current value on the SD memory and send a notification to Mobile phone via BLYNK application.

Step10: If humidity is exceeds the threshold (80\%) then save the current value on the SD memory and send a notification to Mobile phone via BLYNK application.

Step11: If PIR is detected then save "Movement detected" on the SD memory and send a notification to Mobile phone via BLYNK application.

Step12: If Fire is detected then save "Fire detected" on the SD memory and send a notification to Mobile phone via BLYNK application.

Step13: If Gas is detected then save "Gas detected" on the SD memory and send a notification to Mobile phone via BLYNK application.

Step14: If control command was received then turns ON/OFF the corresponding device depending on the command type.

\section{Step15: Go to step 7.}

\section{CONCLUSION}

People are getting more concerned to protect their houses from unauthorized people, the status of the house environment like temperature, humidity, gas leakage and fire. The proposed system can monitor a house by use of sensors that are integrated with an Arduino microcontroller and an Ethernet shield unit. Notification message is used to alert users via mobile phone when a possible intrusion occurs or any abnormal events occurs in the Home environment.

Nowadays, everyone is almost using mobile phone so via using this system, the users will not have to carry additional devices to monitor their house. This system is designed using modularity to become a flexible system that can be add more sensors without change the whole system, only add some sensors to increase systems functionality. So this system is a modular home security system using Ethernet that uses the internet via Blink application function to initiate the communication between the system and the user.

\section{REFERENCES}

Alheraish, A. (2004). Design and implementation of home automation system. IEEE Transactions on Consumer Electronics, 50(4), 10871092.

Butt, M., Khanam, M., Khan, A., \& Khiyal, M. S. H. (2010). Controlling Home Appliances Remotely Through Voice Command. IJACSA) Interna tiona 1 Journal of Adva nced Computer Science a nd Applica tions, Special Issue on Wireless \& Mobile Networks, 3539.

Chitnis, S., Deshpande, N., \& Shaligram, A. (2016). An Investigative Study for Smart Home Security: Issues, Challenges and Countermeasures. Wireless Sensor Network, 8(04), 77.

Gao, L., Wang, Z., Zhou, J., \& Zhang, C. (2016). Design of Smart Home System Based on ZigBee Technology and R\&D for Application. Energy and Power Engineering, 8(01), 13.

Han, J., Choi, C. S., Park, W. K., Lee, I., \& Kim, S. H. (2014). Smart home energy management system including renewable energy based on ZigBee and PLC. IEEE Transactions on Consumer Electronics, 60(2), 198-202.

Lee, K. C., \& Lee, H. H. (2004). Network-based fire-detection system via controller area network for smart home automation. IEEE Transactions on Consumer Electronics, 50(4), 1093-1100.

Liu, Y. (2013). Study on smart home system based on internet of things technology. In Informatics and Management Science IV (pp. 7381). Springer London.

Piyare, R. (2013). Internet of things: ubiquitous home control and monitoring system using android based smart phone. International Journal of Internet of Things, 2(1), 5-11.

Shewale, A. N., \& Bari, J. P. (2015). Renewable Energy Based Home Automation System Using ZigBee. Renewable Energy, 5(3).

Teymourzadeh, R., Ahmed, S. A., Chan, K. W., \& Hoong, M. V. (2013, December). Smart GSM based home automation system. In Systems, Process \& Control (ICSPC), 2013 IEEE Conference on (pp. 306-309). IEEE.

Vanus, J., Vojcinak, P., Martinek, R., Kelnar, M., Machacek, Z., Bilik, P., ... \& Zidek, J. (2016). Building heating technology in Smart Home using PI System management tools. Perspectives in Science, 7, 114-121.

Wu, C. L., Liao, C. F., \& Fu, L. C. (2007). Service-oriented smart-home architecture based on OSGi and mobile-agent technology. IEEE Transactions on Systems, Man, and Cybernetics, Part C (Applications and Reviews), 37(2), 193-205.

Zhang, R., Zou, X., \& Huang, W. (2013). A Smart Home System Design Based on GSM. Communications and Network, 5(01), 25.

Zhai, Y., Liu, Y., Yang, M., Long, F., \& Virkki, J. (2014). A survey study of the usefulness and concerns about smart home applications from the human perspective. Open Journal of Social Sciences, 2(11), 119 . 